\title{
Elimination of thermal instability in precise positioning of Galfenol actuators
}

\author{
Mojtaba Ghodsi ${ }^{a}$, Ashraf Saleem ${ }^{b}$, Abdullah Özer ${ }^{a}$, Issam Bahadur ${ }^{a}$, Khurshid Alam ${ }^{a}$, Amur \\ Al-Yahmadi ${ }^{a}$, Mohammad Hadi Ghodsi ${ }^{c}$, Hamidreza Hoshyarmanesh ${ }^{\text {d, Mohammad Reza }}$ \\ Sheykholeslami ${ }^{\mathrm{e}}$ \\ ${ }^{a}$ Department of Mechanical \& Industrial Engineering, Sultan Qaboos University, Muscat, Oman \\ ${ }^{\mathrm{b}}$ Department of Computer and Electrical Engineering, Sultan Qaboos University, Muscat, Oman \\ ${ }^{\mathrm{c}}$ Department of Mechanical Engineering, Faculty of Engineering, Yazd University, Yazd, Iran \\ ${ }^{\mathrm{d}}$ Health Research Innovation Centre, Department of Clinical Neurosciences, University of Calgary, Canada \\ ${ }^{\mathrm{e}}$ Department of Mechanical Engineering, Faculty of Engineering, Arak University, Arak, Iran
}

ghodsi@squ.edu.om ; moj.ghodsi@gmail.com, Sultan Qaboos University, Al-khoudh, Muscat, Oman

\begin{abstract}
This paper presents a new method to eliminate deviation in positioning caused by coil's heat generation in magnetostrictive actuators. The advantages of the proposed system are compactness, high controllability and high reliability. The actuator package consists of Galfenol as active element and a magnification mechanism combined with a Peltier element or thermoelectric cooler (TEC). By using the temperature sensor, a thermoelectric cooler (TEC) is activated to reduce the temperature of the coil. However, the reduction of temperature by TEC alone is not enough to eliminate the error and controlling of applied voltage is also required. A simple PI controller for coil's current is combined with TEC and by reducing the temperature and current simultaneously, the positioning error is vanished completely.
\end{abstract}

Keywords: Galfenol, Precise positioning, Thermal instability, Peltier element (TEC)

\section{INTRODUCTION}

In last decay, high speed response smart materials like magnetostrictive, piezoelectric and magnetorheological materials play significant roles in mechatronics systems. Terfenol-D [1-4], Galfenol [5-6] and Permandure [7-8] are widely used as actuation elements in the new generations of actuators [9-11] while these materials are widely used in sensors $[12,13]$ and energy saving systems too [14]. Piezoelectric elements like Lead Zirconate Titanate (PZT) have also today numerous applications in electro-mechanical systems such as actuators for medical applications [15-16], health monitoring transducers [17-19]. Magnetorheological materials also are suitable for flexible and tactile actuators, however, low speed response can be counted as their drawback [20-21].

Among these materials, piezoelectric and magnetostricitve (MS) are wieldy used in actuators for precise positioning. Since strains of piezoelectric and magnetostrictive materials are small compared to the shape memory alloys and electro active polymers and magnetorholocical, different magnifying mechanisms were presented $[5,8,16]$. For examples micro amplification mechanism with sub-nanometer resolution in microelectronics industries were developed by micro positioning stages using lever mechanism and piezoelectric. The magnification ration of some of these mechanisms is about 60 which a big displacement of $1.6 \mathrm{~mm}$ was achieved [16]. Although actuators with piezoelectrics driven element has high frequencies, they are not suitable for cryogenic and high humidity or in the other words, harsh environments. Furthermore, piezoelectric needs high voltage. It was shown that Magnetostrictive materials are good candidates to operate in low temperature and low voltage [5]. However, the problem of magnetostrictive material is the heat generated by coil which increases the temperature. Therefore, to keep the desired position of actuator's tip for a period of time which is called "holding time", the coil should be energized continuously. The position deviation proportional to the

Behavior and Mechanics of Multifunctional Materials and Composites 2016, edited by Nakhiah C. Goulbourne, Proc. of SPIE Vol. 9800, 980008 · (c) 2016 SPIE · CCC code: 0277-786X/16/\$18 · doi: 10.1117/12.2222990 
holding time is detected because of coil's generated heat. In this paper a new practical method for eliminating thermal instability in positioning is presented.

\section{PROBLEM STATEMENT}

Internal and external disturbances cause positioning error in MS actuators. Hysteresis and eddy current play significant role in positioning error when the actuators are energized by AC power supply. Any obstacle or resistant load against to the head of actuator can be considered as external disturbances. In this research, main concentration is on the internal disturbances and mainly on positioning error results from the coil's heat. As the coil is energized to keep its position for holding time, the induced magnetic field causes strain in MS material. However, as the current pass through the coil, part of electrical energy is converted to the magnetic energy and part of this energy is dissipated to heat because of Ohmic resistance of coil. Therefore, displacement of MS actuator's head is made by strain of both, magnetic field (Eq. 1) and thermal energy (Eq. 2). In other words, positioning error is proportional to the conducted heat to the magnetostrictive rod.

$$
\varepsilon_{\text {magnetic }}=\frac{\Delta L_{\text {magnetic }}}{L}=\frac{2 B_{0} \Delta B}{E \mu}
$$

Where $B_{0}$ is the magnetic flux density at working point, $\Delta B$ is variable quality of magnetic flux density, $\mu$ and $\mathrm{E}$ are the magnetic permeability and the Young modules of Galfenol, respectively. If the generated heat by the coil causes change in the rod temperature $(\Delta T)$, the thermal strain is calculated by (2).

$$
\varepsilon_{\text {thermal }}=\frac{\Delta L_{\text {thermal }}}{L}=\alpha \Delta T
$$

Where $\alpha$ is thermal expansion coefficient of Galfenol. The total strain is obtained by (3) that its second term made by heat is presented as the positioning error which is equal to the $\alpha \Delta T$.

$$
\varepsilon_{\text {total }}=\varepsilon_{\text {magnetic }}+\varepsilon_{\text {thermal }}=\frac{2 B_{0} \Delta B}{E \mu_{0}}+\alpha \Delta T
$$

As shown in Fig.1, PZT actuator can keep its position for its holding time. However, the Galfenol actuator shows instable behavior because of coil's heat conducted to the Galfenol. Many cooling systems were proposed to remove the generated coil's heat. For examples, Kwak et al. [22], Witthauer et al. [23] and Wang et al. [24] have proposed different air cooling systems and Quanguo Lu et al. [25] and Zhu et al. [26] proposed water cooling systems. Both, air and water cooling systems are suffering from the high cost, difficult sealing and low efficiency. However, in this research Peltier element which is Thermoelectric Cooler (TEC) is exploded which is high response element, cheap, compact and have no insulation problem.

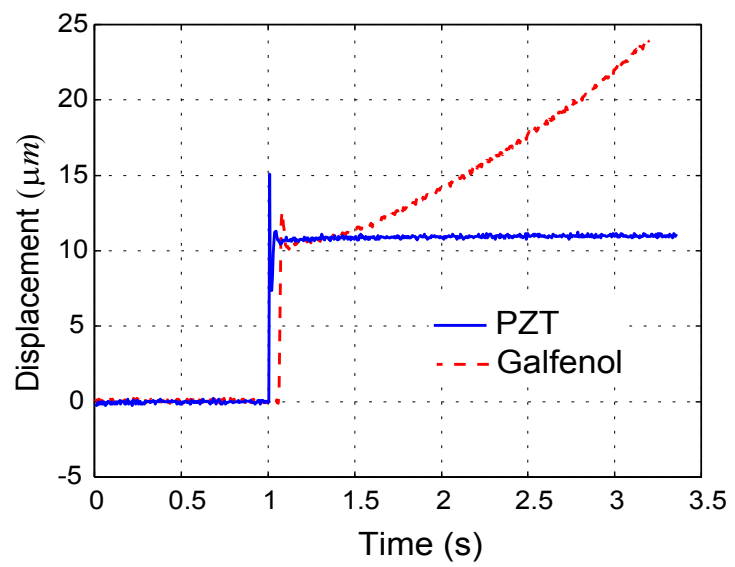

Fig.1: Precise positioning using magnetostrictive and PZT actuator 


\section{PRINCIPLE OF MAGNETOSTRICTIVE ACTUATOR}

Figure 2a shows a schematic configuration of Galfenol actuator. To increase the performance of this actuator, its two main parts make a close magnetic circuit [27-29]. The first part is a Galfenol bar as actuation element and the second part is a magnifying mechanism to amplify strain of the Galfenol. To enhance the performance of magnetic circuit, the magnifying mechanism is made of high permeability magnetic steel [30]. The length of Galfenol bar is $10 \mathrm{~mm}$ and its cross section is square by $1 \mathrm{~mm}$ thickness (Fig. 1b). The maximum strain of annealed Galfenol is about $250 \mathrm{ppm}$. Thickness of magnifying system is $0.8 \mathrm{~mm}$ and energized coil is $\varphi=0.05 \mathrm{~mm}, 240$ turns. Galfenol displacement is transferred to the head by phosphor-bronze spring plate. By energizing the coil, generated magnetic fluxes pass through the Galfenol bar and increase its length. Similar to lever mechanism this enlargement is magnified almost 6 times and large vertical displacement is achievable by head. The initial evaluation shows that $15 \mu \mathrm{m}$ vertical displacement is achievable by this miniature actuator.

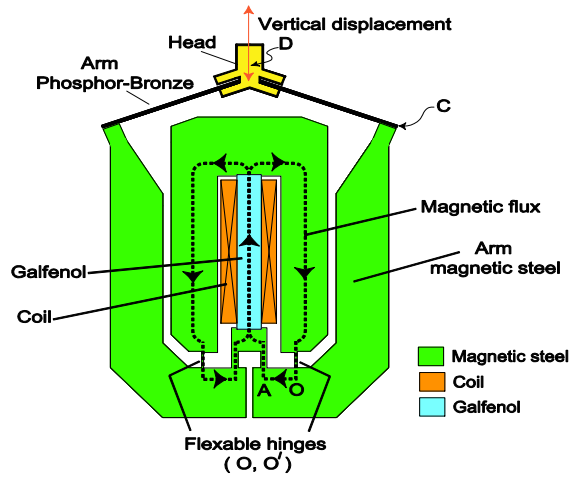

(a)

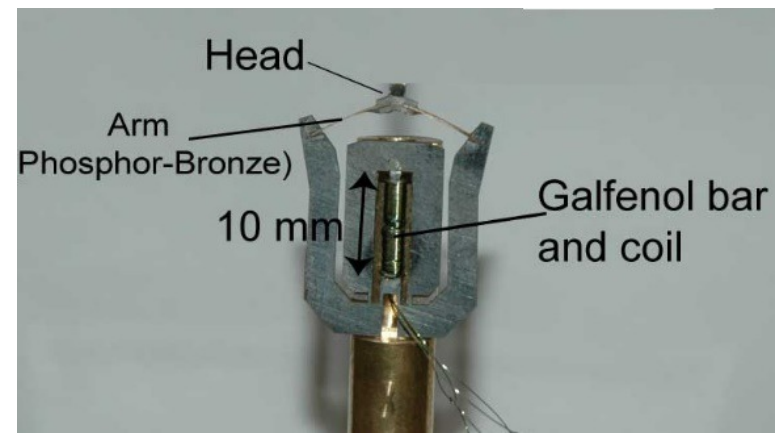

(b)

Fig.2: (a) Schematic of magnetostrictive magnifying system (b) real actuator

\section{POSITION CONTROL SCHEMES AND COOLING SYSTEM}

The closed loop control system is the most appropriate method to reduce the positioning error in actuators. Usually, displacement sensor is exploited as a feedback signal in active control system (Fig. 3). However, to keep the tip of the actuator in a specified position, the coil should be energized by a constant voltage. This constant voltage causes ohmic dissipation and increase temperature of Galfenol. Sometimes, long time positioning increases the coil's temperature to more than $90^{\circ} \mathrm{C}$. In this research a low cost temperature sensor (LM35) is also proposed as a feedback sensor to activate TEC via relay. However, the amount of removed heat by TEC was not enough to cancel the positioning error completely. Consequently, the current passing through the actuator's coil is controlled by a simple PI controller. 


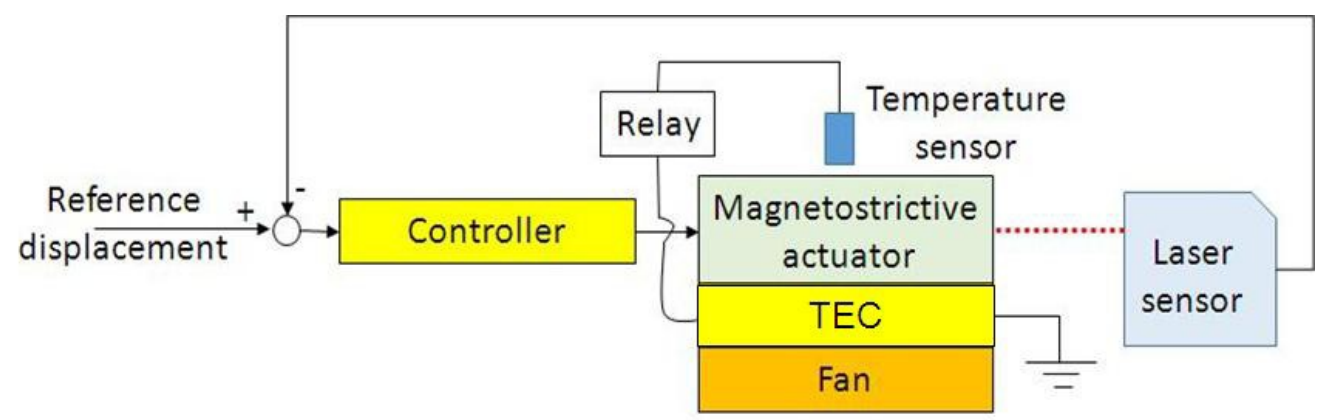

Fig.3: Position closed-loop control block diagram using displacement sensor and TEC.

\section{EXPERIMENTAL SETUP}

Peltier element or thermoelectric cooler (TEC) is a semiconductor that transfer heat from one side to other side in the opposite direction of temperature gradient (from cold to hot). Usually, this element is used in airplane refrigerator for cooling water. To keep the performance of TEC high, temperature diffidence of two sides of TEC should be controlled. Therefore, a copper fin and a fan are attached to the hot side of Peltier element to remove the heat and keep TEC performance high.

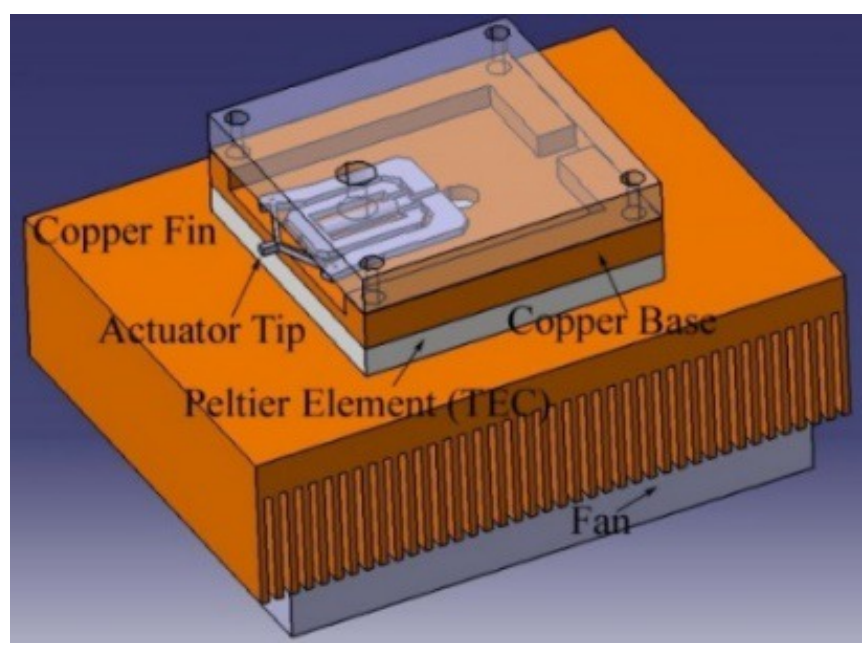

(a)

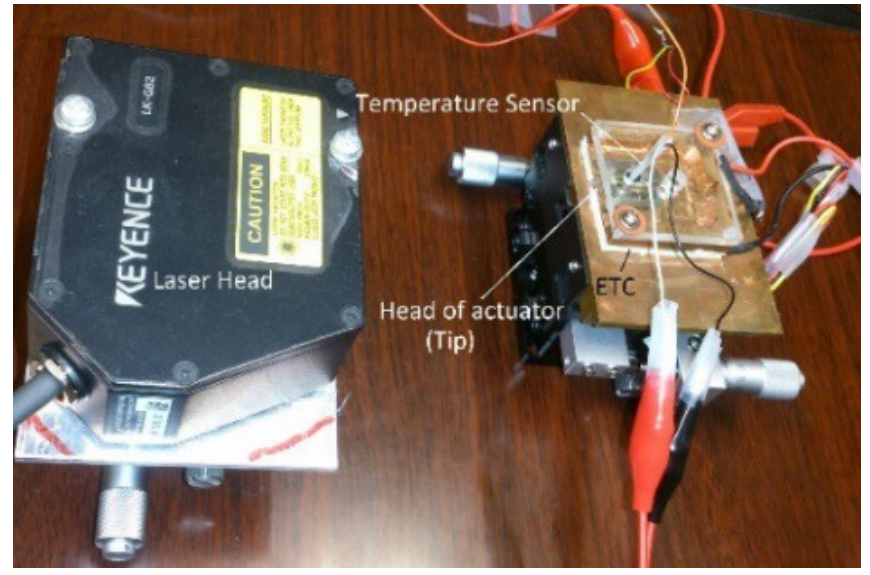

(b)

Fig.4: (a) Schematic configuration of magnetostrictive actuator cooled by Peltier element (TEC) (b) Real photo of cooling system 
To measure the displacement of actuator's tip, bottom side of actuator is fixed on upper side of a thin copper base (40 $\mathrm{mm} \times 40 \mathrm{~mm} \times 5 \mathrm{~mm}$ ). It is well known, that thermal conductivity of copper is around twice of Aluminum thermal conductivity and is about $400 \mathrm{~W} / \mathrm{m} . \mathrm{k}$. As shown in Fig. 4, to remove the heat generated by the exciting coil, cold side of thermoelectric cooler (TEC) is bonded with silicon glue to the bottom side of copper base. The heat is removed from the hot side of TEC by a copper fin attached by a high-speed fan. The temperature sensor (LM35) is used to measure coil's temperature. Upper side of actuator is covered by transparent plexy glass which is a good thermal isolator with low thermal conductivity. As it was explained in previous sections, the Galfenol is ductile and EDM is the best way to cut it. The magnifying part is a lever mechanism and its stiffness should be high enough to amplify the small displacement of Galfenol bar with minimum power loss. Moreover, the material should be magnetic material. Therefore, the material is special grade of magnetic steel with high permeability. To manufacture the magnifying mechanism and head, wire EDM technique is exploited which is fast and accurate enough. Keyance laser sensor (LK-G82) is used to measure the displacement on the tip of actuator when it is energized. Our idea for reducing the thermal effect is removing the heat from the magnetostrictive material using Peltier element.

\section{RESULTS AND DISCUSION}

The relationship between tip displacements of actuator without controlling the coil's current is shown in Fig. 5. By energizing the actuator with step constant voltage, the vertical displacement, coil's temperature, current passing through the coil are measured. As shown in Fig. 5, by applying a 6V constant voltage, a current of $0.8 \mathrm{~A}$ is passing through the coil which causes $5 \mu \mathrm{m}$ displacement in the tip of the actuator. It is found that, by keeping the current for only 3 seconds, the coil's temperature reaches to $27.5^{\circ} \mathrm{C}$ from its initial temperature of $24.5^{\circ} \mathrm{C}$. Because of high thermal coefficient of Galfenol $\left(\alpha=11.7 \mathrm{ppm} /{ }^{\circ} \mathrm{C}\right)$ a giant error displacement of about $110 \mu \mathrm{m}$ is produced. Current reduction is predictable because of higher coil's resistor by constant applied voltage. These measurements are repeated by $4 \mathrm{~V}$ in Fig. 6 and the same behavior is verified.

By using closed-loop control scheme presented in Fig. 4, the thermal disturbance made by coil's heat is eliminated. In this control system, the TEC is switched $\mathrm{ON}$ as the temperature is more than $25{ }^{\circ} \mathrm{C}$. Based on the dynamic behavior, resonance frequency of the actuator [5, 31] and model identification [32-35] of actuator, a Proportional-Integral (PI) controller is designed to eliminate the effect of heat. After precise tuning, the coefficients of PI controller by $\mathrm{K}_{\mathrm{p}}=3.25$ and $\mathrm{K}_{\mathrm{i}}=40.52$ is exploited. As shown in Fig. 7, the coil is energized to position the tip at $5 \mu \mathrm{m}$. To keep the position of 5 $\mu \mathrm{m}$ for 2 seconds, the applied voltage is controlled in a way that reduces the current passing through the coil while the TEC is turned ON.
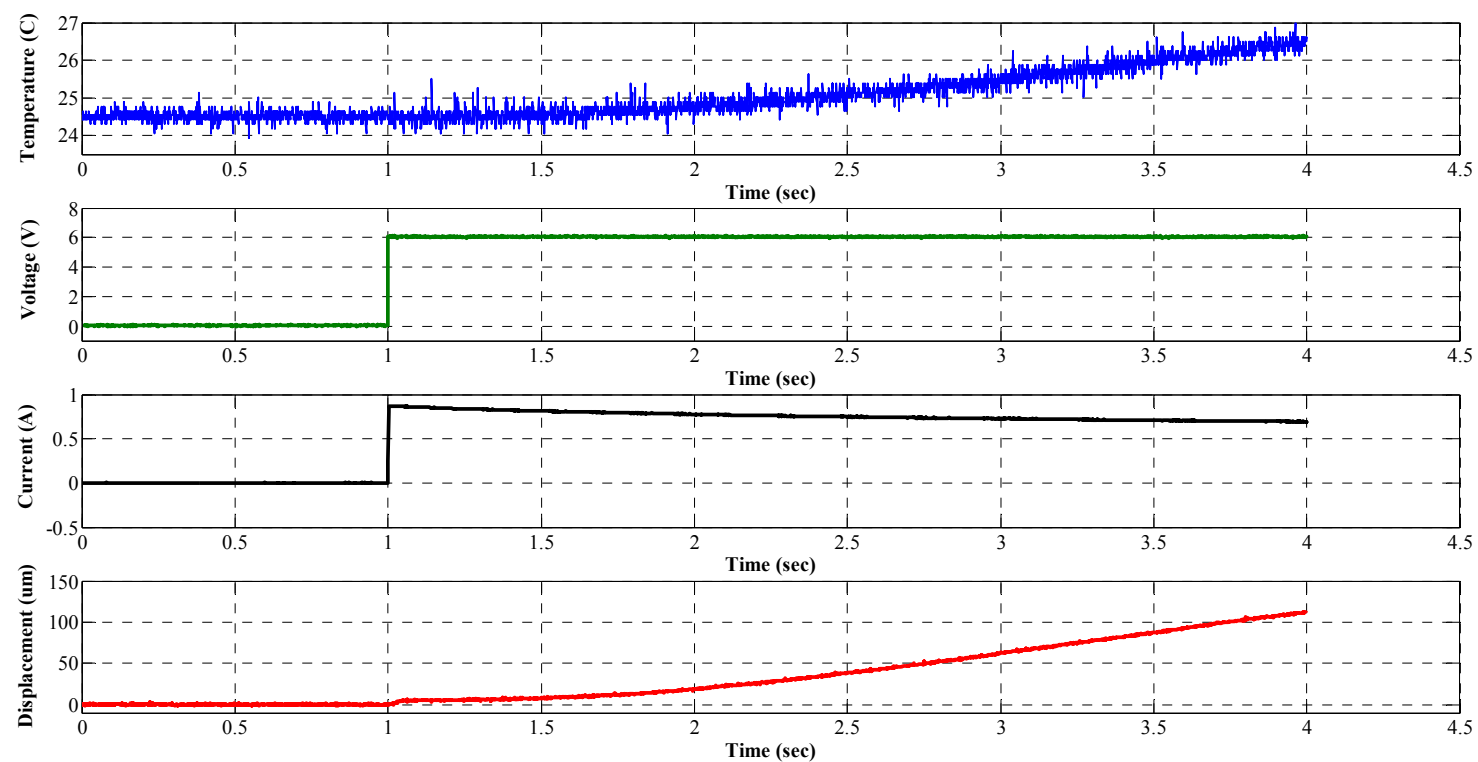

(a) 


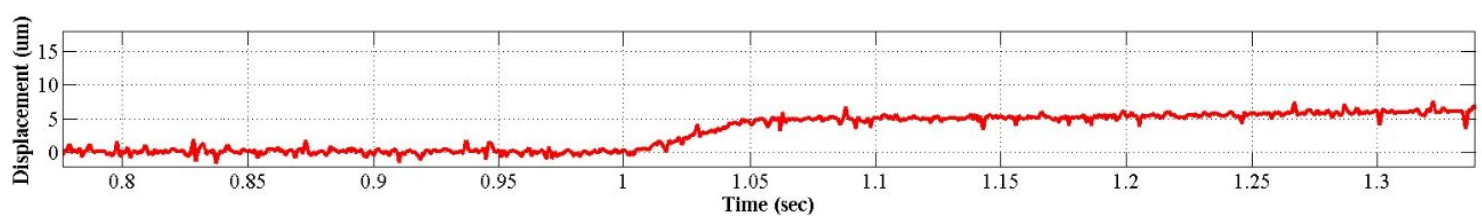

(b)

Fig.5: (a) Temperature, voltage, current and displacement vs. time by energizing constant voltage of 6V (b) Zoom of displacement
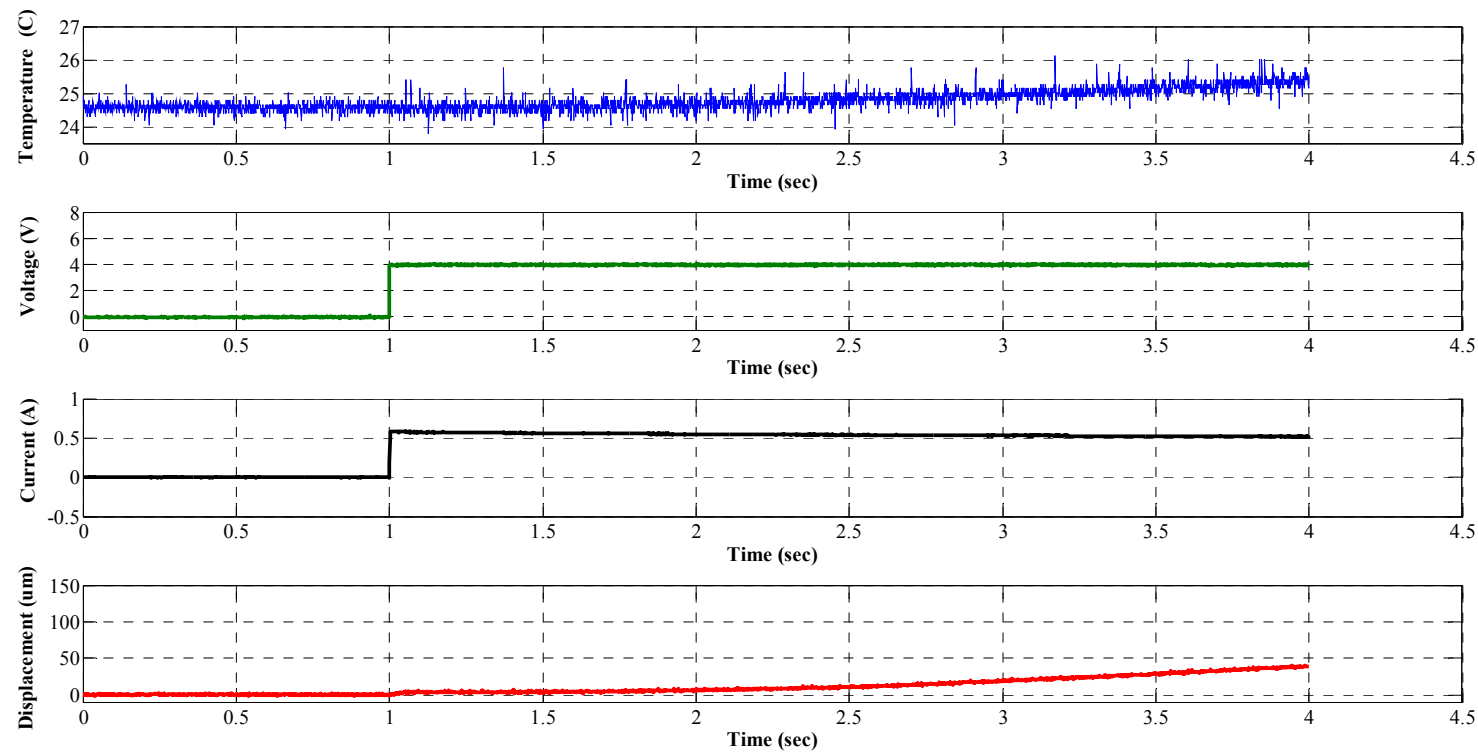

Fig.6: Temperature, Voltage, Current and Displacement vs. time by energizing constant voltage of 4V 

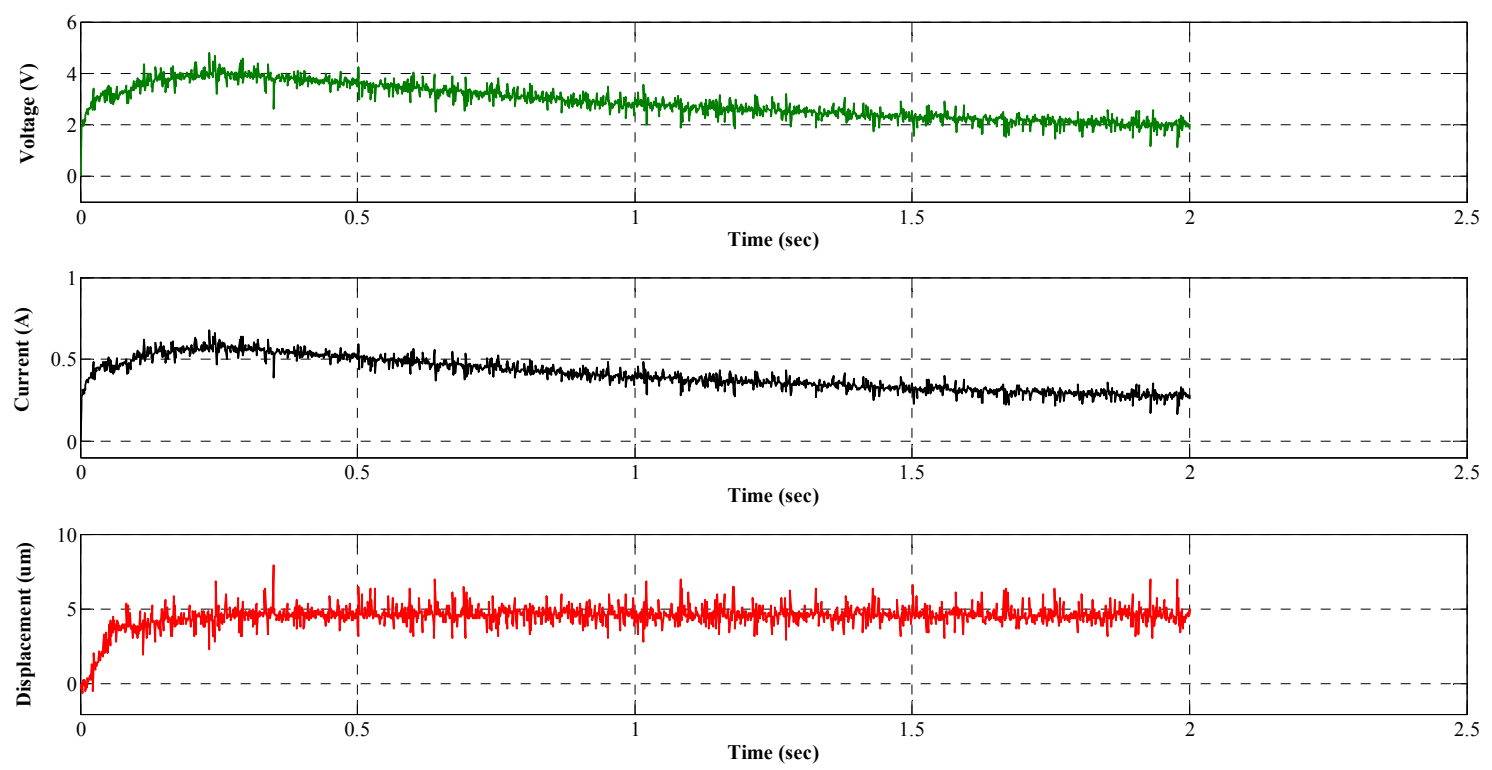

Fig.7: Displacement control using displacement and temperature sensors with turning the TEC

\section{CONCLUSIONS}

In summary, a closed-loop control technique combined by a cooling system for precise positioning of Galfenol actuator was presented. In this technique, both, displacement and temperature sensors were exploited. It was shown that the temperature rise because of continuous coil energizing is the main reason of positioning error. By using the temperature sensor, a thermoelectric cooler (TEC) was activated to reduce the temperature of the coil. However, the reduction of temperature by TEC alone was not useful and controlling of applied voltage was also required. A simple PI controller was combined to TEC and by reducing the temperature and current, the positioning error was eliminated completely.

\section{Acknowledgment}

This work was done under the projects number (IG/ENG/MIED/15/02) and (IG/ENG/ECED/15/01) which were funded from the internal grants of Sultan Qaboos University in Oman.

\section{References.}

[1] Soheil Talebian, Yousef Hojjat, Ghodsi, Mojtaba, Mohammad Reza Karafi and Shahed Mirzamohammadi, "a Combined Preisach-Hyperbolic Tangent Model for Magnetic Hysteresis of Terfenol-D," Journal of Magnetism and Magnetic Materials, Vol.396, pp. 38-47 (2015).

[2] Soheil Talebian, Yousef Hojjat, Mojtaba Ghodsi, Mohammad Reza Karafi "Study on Classical and Excess Eddy Currents Losses of Terfenol-D” Journal of Magnetism and Magnetic Materials, Vol.388, pp. 150-159 (2015).

[3] Soheil Talebian, Yousef Hojjat, Mojtaba Ghodsi, Mohammad Reza Karafi, "Study on magnetic hysteresis of Terfenol-D using new hybrid model”: Key Engineering Materials Vol.605, pp 519-522, (2014).

[4] Mojtaba Ghodsi, Hamid Rajabzadeh, Nasser Hosseinzadeh, Nader Garjasi Varzeghani, Yousef Hojjat, Soheil Talebian, M. Sheykholeslami, Amer Al-Yahmadi and Abdullah Özer, "Development of Gasoline Direct Injector Using Giant Magnetostrictive Materials" IEEE Industry Applications Magazine, (2016).

[5] Mojtaba Ghodsi, Toshiyuki. Ueno, Mehdi Modabberifar: "Quality Factor, Static and Dynamic Responses of Miniature Galfenol Actuator at Wide Range of Temperature" International Journal of Physical Sciences, Vol. 6(36), pp 8143-8150 (2011). 
[6] Mojtaba Ghodsi, Toshiyuki Ueno, S.K Chee, Takashi Yano and Toshiro Higuchi "Miniature Iron-Gallium Actuator with Displacement Magnifying Mechanism” IEEE International conference, Intermag2008, pp. 841-843, Spain (2008).

[7] Mohamadreza Sheykholeslami, Yousef Hojjat, Mojtaba Ghodsi , M Zeighami , Keyvan Kakavand, " Effect of magnetic field on Mechanical properties in Permandur", Materials Science \& Engineering A" 651, pp. 598-603 (2016).

[8] Mojtaba Ghodsi, Toshiyuki Ueno, Toshiro Higuchi: "Novel Magnetostrictive Bimetal Actuator Using Permendur" Advanced Materials Research, Vols. 47-50, pp. 262-265 (2008).

[9] Mohamadreza Sheykholeslami, Yousef Hojjat, Mojtaba Ghodsi, Keyvan Kakavand and Simone Cinquemani "Investigation of $\Delta \mathrm{E}$ Effect on Vibrational Behavior of Giant Magnetostrictive Transducers" Shock and vibration, ID 478045,9 (2015).

[10] Mohammad Reza Karafi, Mojtaba Ghodsi, Yousef Hojjat, "Development of Magnetostrictive Resonant Torsional Vibrator" IEEE Transactions on Magnetics, Vol. 51, No. 9, 4003608 (2015).

[11] Mohammad Reza Karafi, Yousef Hojjat, Farokh Sasani, Mojtaba Ghodsi, "A Novel Magnetostrictive Torsional Resonant Transducer" Sensors and Actuators $A$ Vol. 195, pp.71-78 (2013).

[12] Mojtaba Ghodsi, Shahed Mirzamohammadi, Yousef Hojjat, Soheil Talebian, Mohamadmorad Sheikhi, Abdullah Ozer, Amur Al-Yahmadi "Analytical, Numerical and Experimental Investigation of a Giant Magnetostrictive (GM) Force Sensor” Sensor Review, Vol. 35 No. 4, pp. 357-365 (2015).

[13] Fernando Seco, Jose Miguel Martin, Antonio Ramon Jimenez, Leopoldo Calderon, "A high accuracy magnetostrictive linear position sensor" Sensors and actuators A 123-124, pp. 216-223 (2005).

[14] Mojtaba Ghodsi, Toshiyuki Ueno, Hidekazu Teshima, Hosei Hirano and Toshiro Higuchi and Eric Summers: "ZeroPower Positioning Actuator for Cryogenic Environments by Combining Magnetostrictive Bimetal and HTS" Sensors and Actuators A, Vol.135, pp. 787-791 (2007).

[15] Hesam Sadeghian, Yousef Hojjat, Mojtaba Ghodsi, Mohammad Reza Sheykholeslami, "An approach to design and fabrication of a piezo-actuated microdroplet generator" International Journal of Advanced Manufacturing Technology, DOI 10.1007/s00170-013-5371-5 (2013).

[16] Takashi Yano, Sze Keat Chee, Kazuo Yakuwa, Saburo Harada, Toshiro Higuchi "A New Type of Mechanical Transformer with High Stroke Magnification Ratio" Actuator2008, pp. 71-74, Germany (2008).

[17] Hamidreza Hoshyarmanesh, Naser Nehzat, Mehdi Salehi , Mojtaba Ghodsi , Hong-Sub Lee and Hyung-Ho Park "Thickness and thermal processing contribution on piezoelectric characteristics of $\mathrm{Pb}(\mathrm{Zr}-\mathrm{Ti}) \mathrm{O} 3$ thick films deposited on curved IN738 using sol-gel technique" Journal of Materials: Design and applications, DOI,10.1177/1464420714568787 (2015).

[18] Hamidreza Hoshyarmanesh , Naser Nehzat, Mehdi Salehi , Mojtaba Ghodsi , "X-ray Diffraction Measurement of Residual Stress-Strain in Sol-Gel Grown Lead Zirconate Titanate Thick Films Deposited on Nickel-Based Supper Alloy Substrate" Journal of Mechanical science and Technology, 29 (2) 715-721 (2015).

[19] Hamidreza Hoshyarmanesh , Naser Nehzat, Mehdi Salehi, Mojtaba Ghodsi , Hong-Sub Lee and Hyung-Ho Park "Piezoelectric Transducers on Curved Dispersive Bending Wave and Poke-Charged Touch Screens" Materials and Manufacturing Processes, 29:7, 870-876, DOI: 10.1080/10426914.2014.921710 (2014).

[20] Aref Naimzad, Yousef Hojjat, Mojtaba Ghodsi, "Porous and Nonporous Film-Shaped Magnetorheological Nanocomposites: Dielectric and Electrical Properties" Smart Materials Research, Article ID 764303, (2014).

[21] Aref Naimzad, Yousef Hojjat, Mojtaba Ghodsi, "Comparative Study on Mechanical and Magnetic Properties of Porous and Nonporous Film-Shaped Magnetorheological Nanocomposites based on Silicone Rubber" International Journal of Innovative Science and Modern Engineering, Vol.-2, Issue-8, July 15, (2014).

[22] Yong-kil Kwak, Sun-Ho Kim and Jung Hwan Ahn, "Improvement of positioning accuracy of magnetostrictive actuator by means of built-in air cooling and temperature control" International Journal of Precision Engineering and Manufacturing, Vol.12, No. 5, pp. 829-834, (2011).

[23] Adam Witthauer, Gap-young Kim, LeAnn Faidly, Qingze Zou, Zhihuua Wang, "Design and characterization of a flextensional stage based on Terfenol-d actuator" International Journal of Precision Engineering and Manufacturing, Vol.15, No. 1, pp. 135-141, (2014).

[24] Zhihua Wang, Adam Witthauer, Qingze Zou, Gap-Yong Kim and LeAnn Faidley, "Control of a Megnetostrictive actuator-Based Micromachining system for optimal high-spped microforming process" IEEE/ASME Transactions on Mechatronics, Vol. 20, No.3, pp. 1046-1055, (2015). 
[25] Quanguo Lu, Zhon Min, Cao Qinghua, Chen Jing, Chen Dingfang, Yan Jianwu, “Integrated Optimized Design of GMA with double water-cooling cavums" IEEE International Conference on Mechanic Automation and Control Engineering (MACE),pp. 3562-3565,(2010).

[26] Yuchuan Zhu, Liang Ji, "Theoretical and experimental investigations of the temperature and thermal deformation of a giant magnetostrictive actuator" Sensors and actuators A, 218, pp. 167-178, (2014).

[27] Mojtaba Ghodsi, "Optimization of Mover Acceleration in DC Tubular Linear Direct-Drive Machine Using Response Surface Method" International Review of Electrical Engineering, Vol. 10, No. 4, pp. 492-500 (2015).

[28] Mojtaba Ghodsi, Toshiyuki Ueno, Hidekazu Teshima, Hosei Hirano and Toshiro Higuchi "Numerical Modeling of Iron Yoke Levitation Using the Pinning Effect of High Temperature Superconductor" IEEE Transactions on Magnetics, Vol. 43, No. 5, pp. 2001-2008 (2007).

[29] Mojtaba Ghodsi, Toshiyuki Ueno, Hidekazu Teshima, Hosei Hirano and Toshiro Higuchi: "The Characteristics of Trapped Magnetic Flux inside Bulk HTS in the Mixed- $\mu$ Levitation System" Physica C: Superconductivity and its Applications, Vol.445, pp. 343-346 (2006).

[30] Mojtaba Ghodsi, Mohammadreza Loghmanian "Effect of forging on ferromagnetic properties of low-carbon steel" IEEE International conference ICMSAO 2011, Kuala Lumpur, Malaysia (2011).

[31] Abdullah Özer, Mojtaba Ghodsi, Akio Sekiguchi, Ashraf Saleem and M. Nasser Al-Sabari "Design and Experimental Implementation of a Beam-type Twin Dynamic Vibration Absorber for a Cantilevered Flexible Structure Carrying an Unbalanced Rotor: Numerical and Experimental Observations" Shock and vibration, Article ID 154892 (2015).

[32] Tarek Tutunji and Ashraf Saleem, "A methodology for identification and control of electro-mechanical actuators, MethodsX" Vol. 2, 219-231. (2015).

[33] Ashraf Saleem, Rateb Issa, and Tarek Tutunji, "Hardware-In-the-Loop for on-line identification and control of threephase squirrel cage induction motors" Simulation Modelling Practice and Theory 18, 277-290, (2010).

[34] Ashraf Saleem, Bashar Taha, Tarek Tutunji, Ahmed Al-Qaisia, "Identification and cascade control of servopneumatic system using Particle Swarm Optimization” Simulation Modelling Practice and Theory, Volume 52, pp 164-179 (2015).

[35] Ashraf Saleem; Saber Abdrabbo; Tarek Tutunji, “On-line identification and control of pneumatic servo drives via a mixed-reality environment" International Journal of Advanced Manufacturing Technology, Volume: 40, Issue: 5-6, pp: 518-530 (2015). 